\author{
انواع ديدان الوحل \\ ( Oligochaeta: Naididae: Tubificinae ) \\ من بيئات مائية مختلفة ضمن مدينة بغداد / العراق \\ افراح محد علوان** \\ هيفاء جواد جويرث*

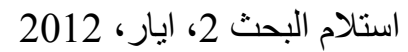 \\ قبول النشر 19، حزيران، 2012
}

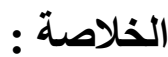

استهدفت الداسة الحالية بصورة رئيسة عزل وتثخيص أنواع ديدان الوحل تحت العائلة

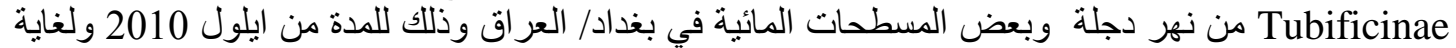

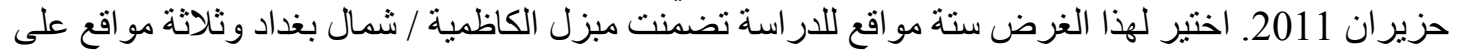

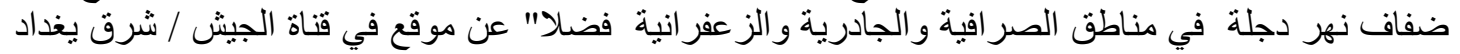

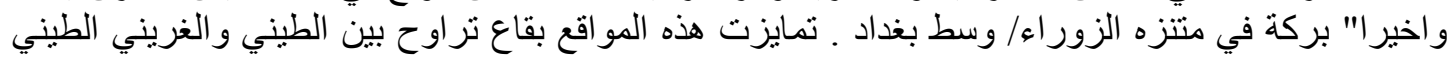

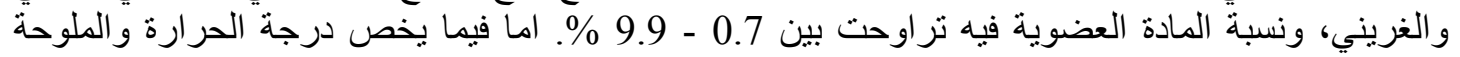

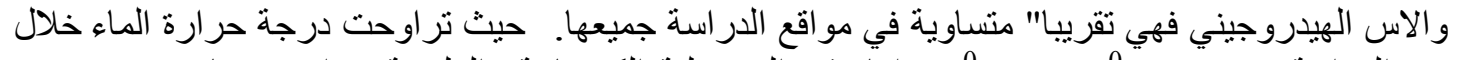

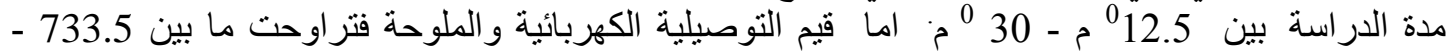

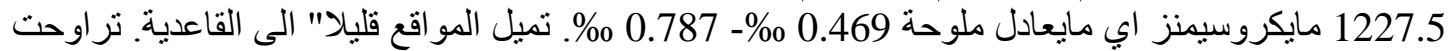

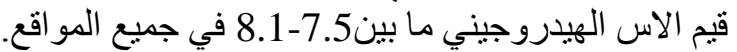

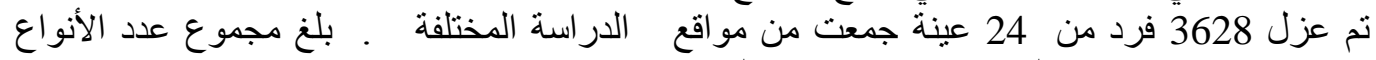

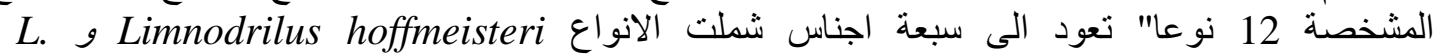
و Tubifex tubifex و L. silvani , L.udekemianus و L. profundicola g claparedeianus Psammoryctides , P. hammoniensis و Potamothrix. bavaricus , Branchiura sowerbyi . Rhyacodrilus coccineus و Monopylephorus irroratus g moravicus

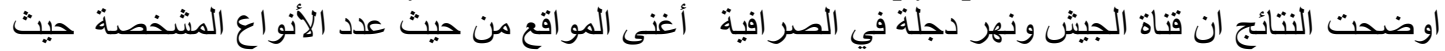

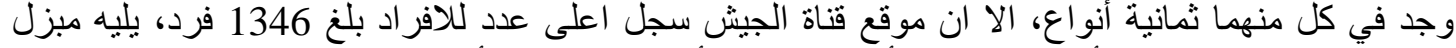

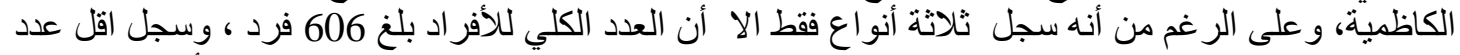

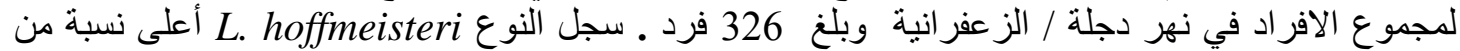

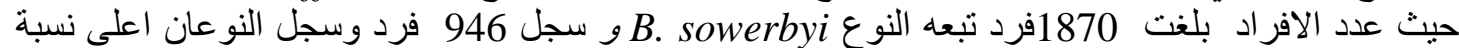

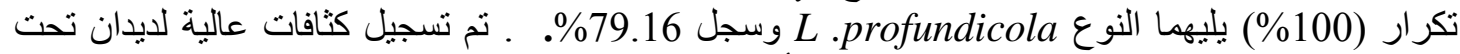

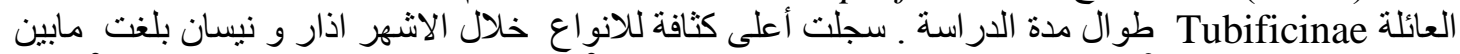

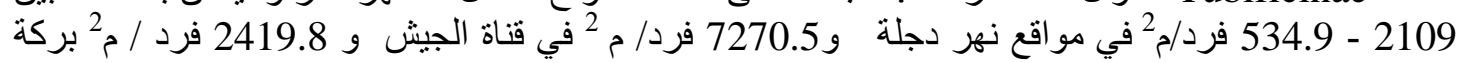

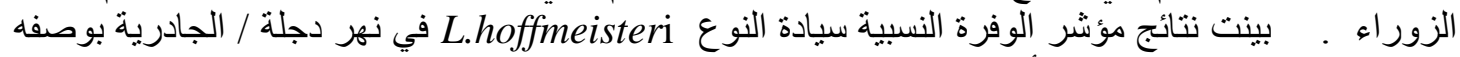

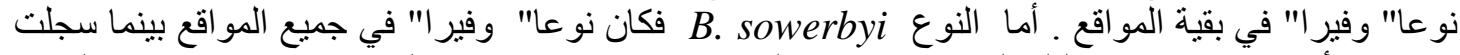

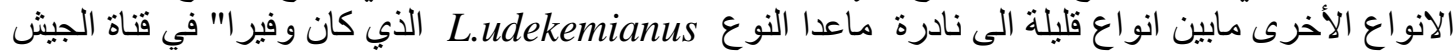

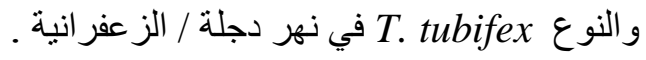

Tubificinae Naididae الكلمات المفتاحية:ديدان الوحل ـ قليلة الاهلاب المائية

بعض الانواع بين النباتات ـ تقتات معظم

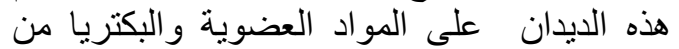

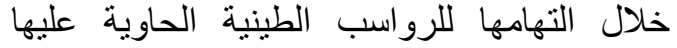

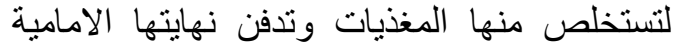
غالبا" بالقاع داخل انابيب او انفاق تنبنيها من الدقائق

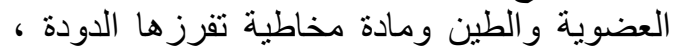

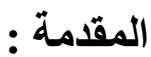

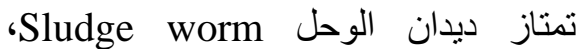

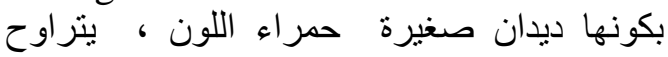

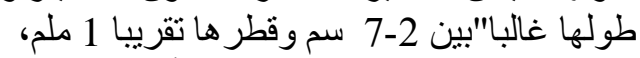

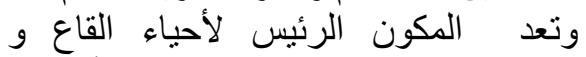

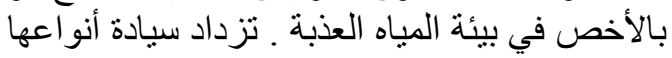
في المناطق الضحلة الطينية و الملوثة، وقد توند توند 


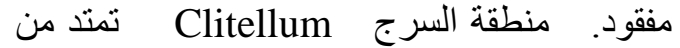
الحلقات التاسعة ( IX ) الى الحلقة الحادية عشرة

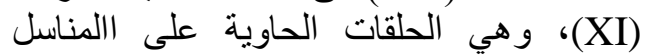
gonads Tubificidae بالطريقة الجنسية غالبا"، و البعض الجنان

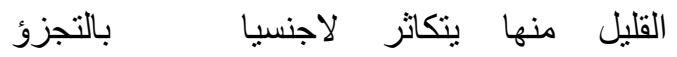
الانواع Fragmentation الانواع عذريا Parthenogenetically او بعطية اخصاب ذاتي Self- fertilization الانتاج افر اد جديدة دون الحاجة لوجي لود القرين و مثال

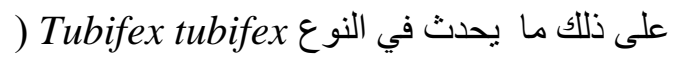

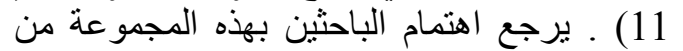

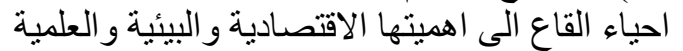

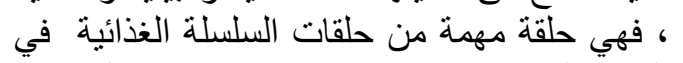

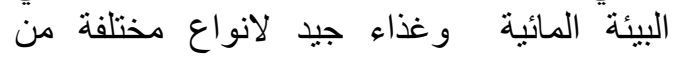

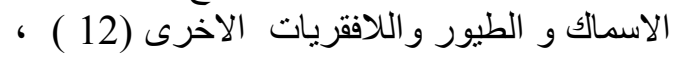

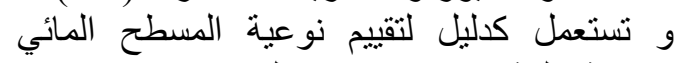

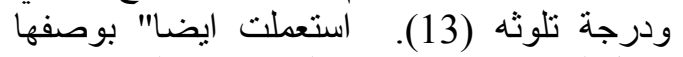

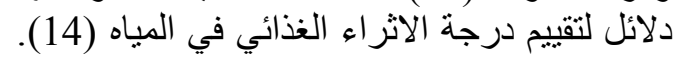

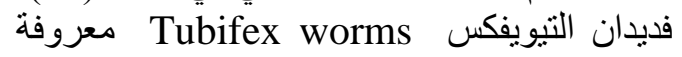
في تجارة الحيوانات Pet trade بوصفئات

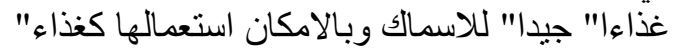
حيا" في احواض تربية الاسماك التجارية

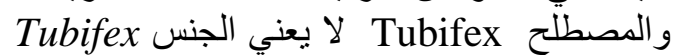
ولكنه يثير الى مجموعة من أنواع تحت العنائلة Lumbriculidae و العائلة Tubificinae

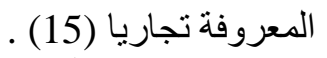

استهدفت الدراسة الحالية عزل ونة ونتخيص

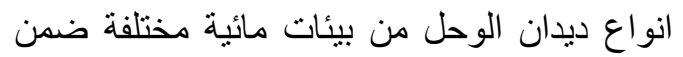

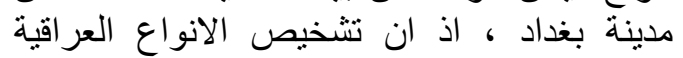

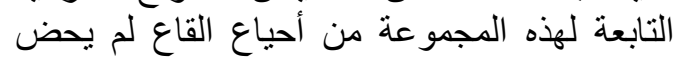

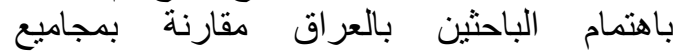

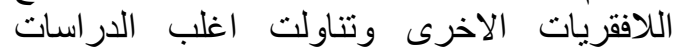

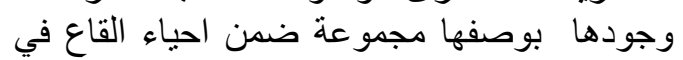

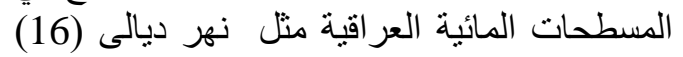
ونهر الفرات ( , 17 و 18و الفئرة 19) وفي نهر دجلة

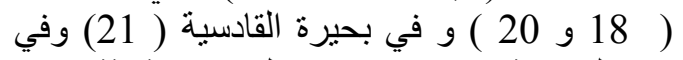

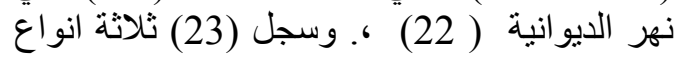

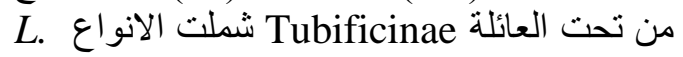
و L. profundicola g claparedianus / Peloscolex velutinus

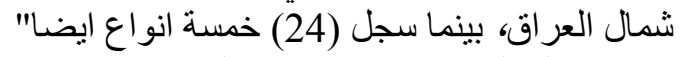

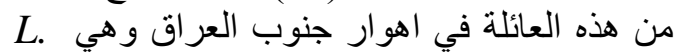
B. و L.hoffmeisteri g claparedeianus Tubifex و L. maumeensis ، sowerbyi tubifex

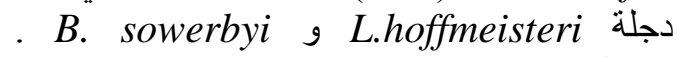

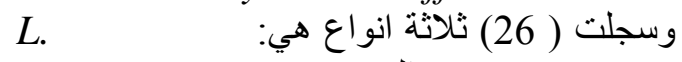
Embolocephalus والنو انوع profundicola
تاركة مؤخرة الجسم تتمرجح في الماء لغرض

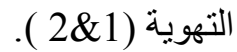

ان وجود و انتشار قليلة الاهلاب المائية يناثر بعدة

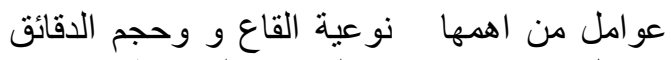

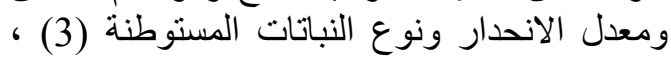

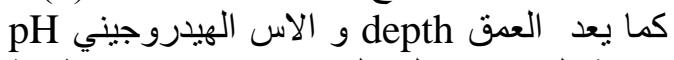

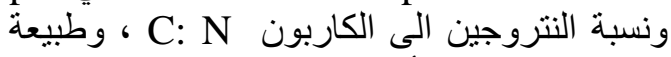

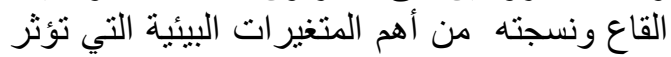

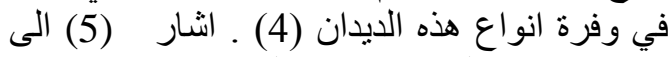

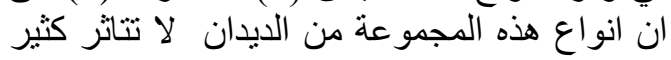

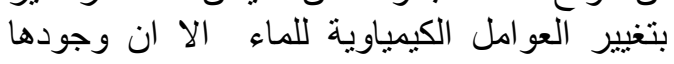

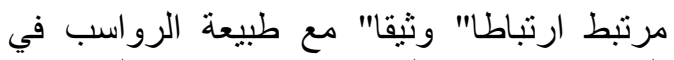

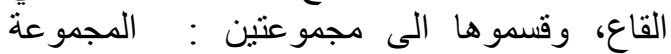
Pilophilous المحبة للقاع الطيني Limnodrilus مثل النوع assemblage shoffmeisteri

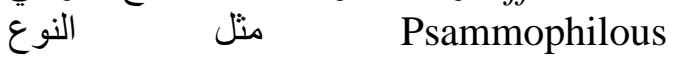
. Psammoryctides barbatus جمعت انواع ديدان الوحل سابقا" بعائلة Tubificidae Subclass Oligochaeta العاس العلاقات التطورية Phylogeny بالاعتماد على تقانة البيولوجي الجزئئي اثبتئ وجود ارتباط جيني قوي بين العائلتين Naididae

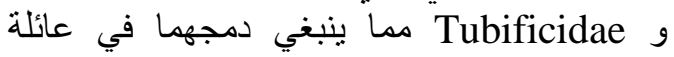

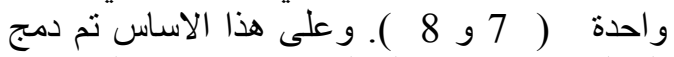

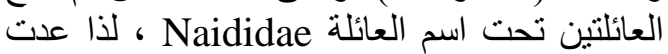

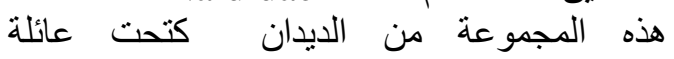
Nubificinae

تتمايز الحلقة ل الاولى في انواع تحت العائلة Tubificinae

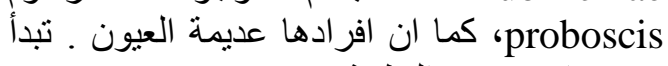

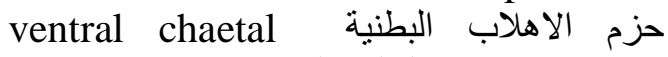
bundles محدودة العدد في الحزمة الونة الواحدة، و ذات ذات نهاية

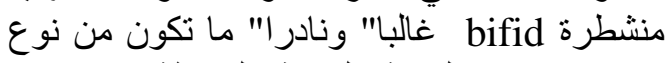
الاهلاب ذات النهاية المدببة البسيطة لونابة Dorsal تبدأ حزم الاهلاب الظهرية النيطة pointed ومن الحلقة الثانية ايضا"،

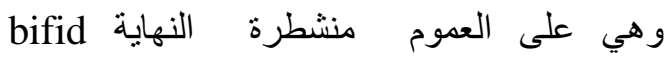

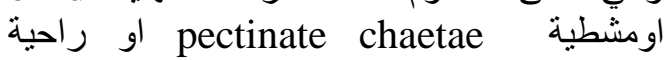
palmate chaetae

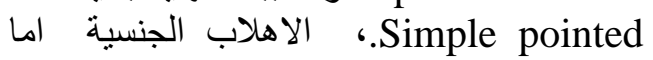

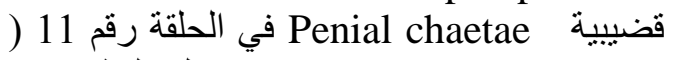

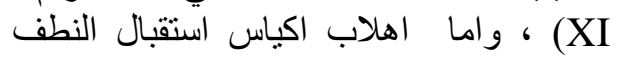
Spermathecal يكون النوعان موجودين في النوع نفسه او كليهما 
فحصها من قبل المركز الوطني للموارد المائية /

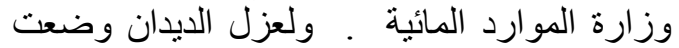
عينة الرواسب في غربال سعة فتحاته 0.5 ملم المانية و غسلت العينة عدة مر ات للتخلص من التهنة الطين بماء

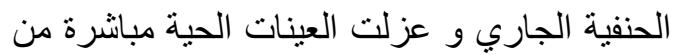
خلال نشر كمية من الراسب الطيني في في صنية الطنية بيضاء اللون مع قليل من الماء ومن ثن الرماء التقاطها

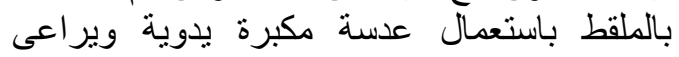

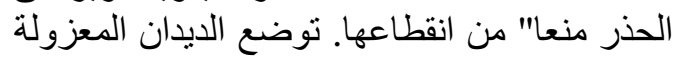

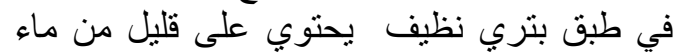

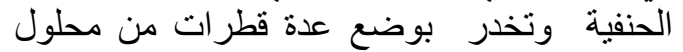

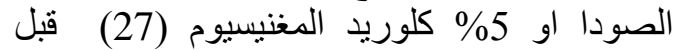

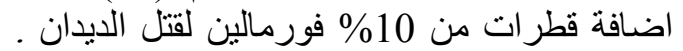

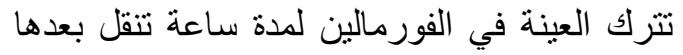

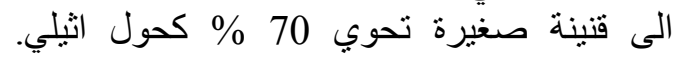

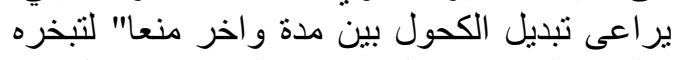

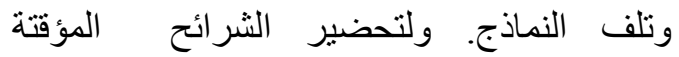

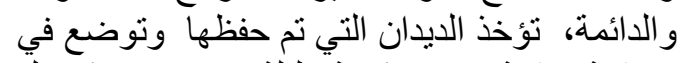

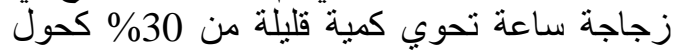

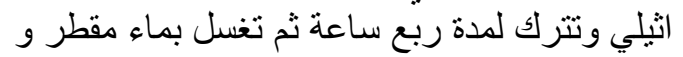

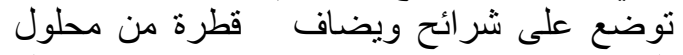

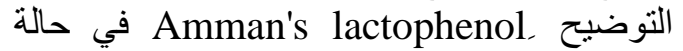
الشرائح المؤقتة و Polyvinyl Lactophenol

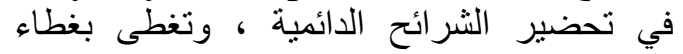

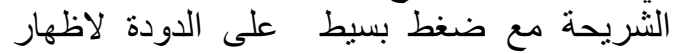

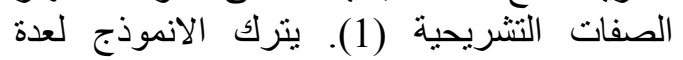

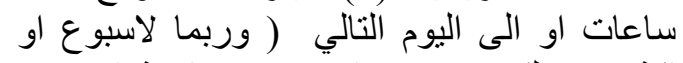

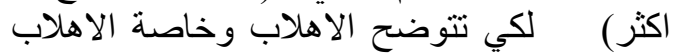

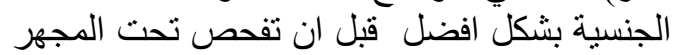

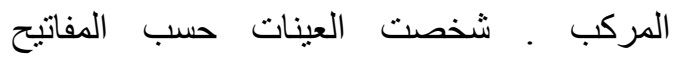
التصنيفية ( 1 و6). عند الانتهاء من عملية العزل و التشخيص تسجل

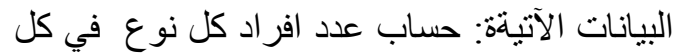

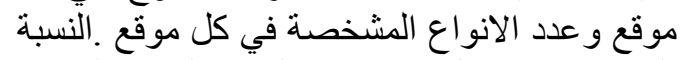

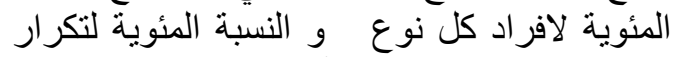

$$
\begin{aligned}
& \text { النوع في العينة تحسب كالآتي: } \\
& \text { نسبة تكر ار النوع س في الّينة }
\end{aligned}
$$

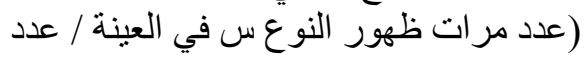

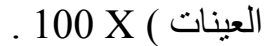

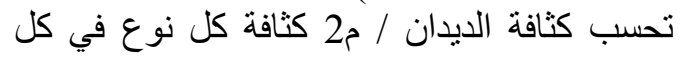
موقع و مؤشر الوفرة النسبية اعتمادا على المعادلة فئلة

$$
\mathrm{Ra}=(\mathrm{N} X 100) / \mathrm{Ns}
$$

$$
\text { التي وردت في (28)، اذن الني }
$$

$$
\text { إذ إن: Ns = عدد افراد النوع في العينة. }
$$$$
\text { Ns }
$$

ولقد عبر عن النتائج باستعمال النسبة المئوية وكما

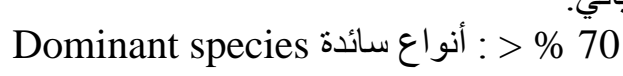

هور و النوع velutinus هور الحويزة جنوب العراق.

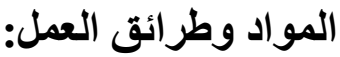

أختير للاراسة الحالية ستة مواقع بيئة مائية

مختلفة داخل مدينة بغداد ( الخارطة 1 ) و شملت ملته

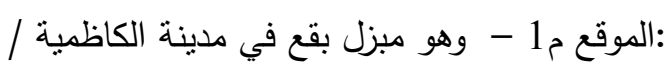

شمال بغداد بالقرب من مصبه في نهر دجلة.

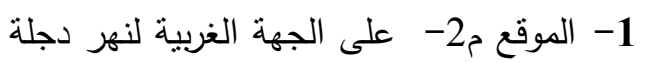

في منطقة الصرافية تحت جسر الصرافية.

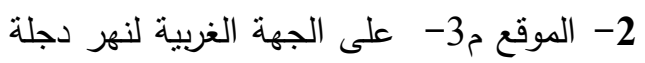

في منطقة الجادرية تحت جسر الجادرية

بالقرب من جزيرة الاعراس السياحية.

3- الموقع م4- على الجهة الثرقية لنهر دجلة

في منطقة الزعفرانية .

4- الموقع م5- على امنداد قناة الجيش شرق لرعربه

بغداد

5- الموقع م6- بحيرة اصطناعية في متنزه

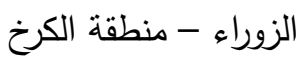

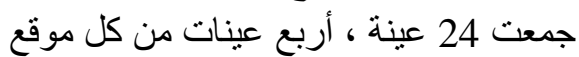

وبو اقع اربعة مكررات لكل عبنة، و و للمدة كلينات

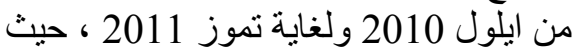

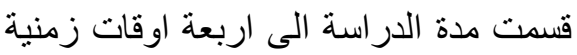

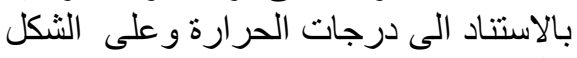

الآتي:

1- الاثشهر من ايلول - تشرين الثاني 2010

2- الاشهر من كانون الاول 2010- شبرل

2011

3- الاشهر آذار ونيسان 2011

4- الاشهر آيار و حزيران 2011

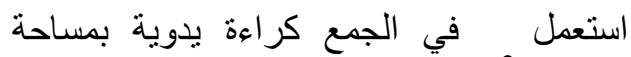

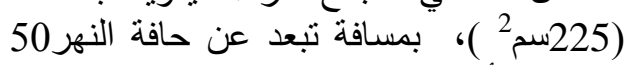

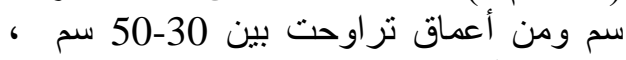

وضعت العينات في حاويات بلاستيكية مع كمية

من ماء النهر ونقلت الى المختبر.

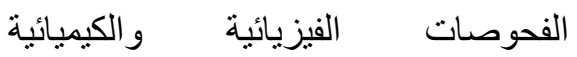

Physical and chemical tests المدروسة حاليا تم الحصول عليها من قبل دائرة المباء

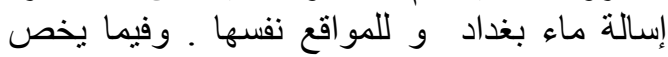
نسجة التربة المئوية للمادة العضوية للمواقع المدروسة فتم 


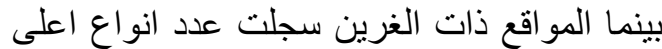

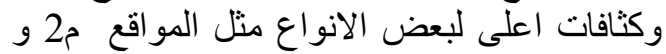

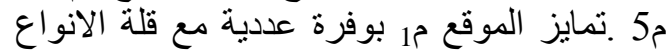

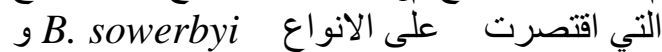
بالاضافة الى اعداد قليلة من L. hoffmeisteri

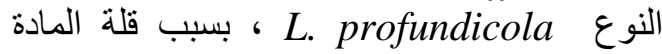

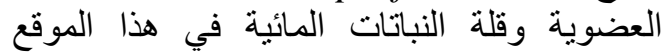
فضلا" عن كونه ذا قاع طيني ( جدولَ، 2 فئس ).

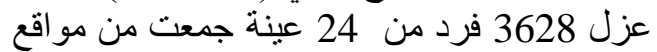

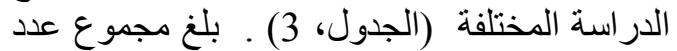
الأنواع المشخصة 12 نوعا" تعود الى الى سبعة العيدة

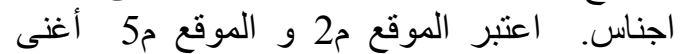

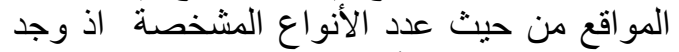

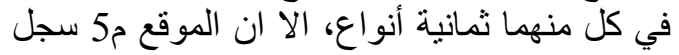

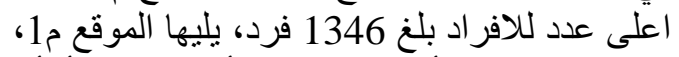

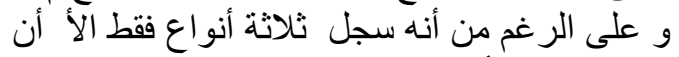

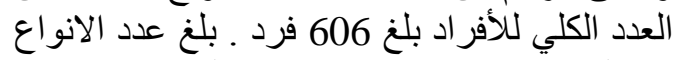

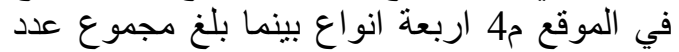

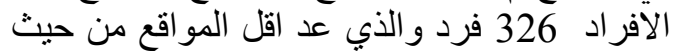

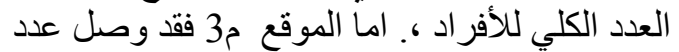

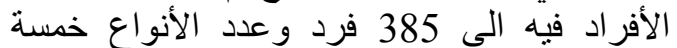

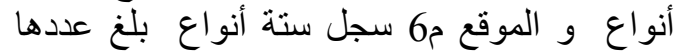
الكلي 597 فرد.

وفيما يخص الانواع المشخصة فقد سجل

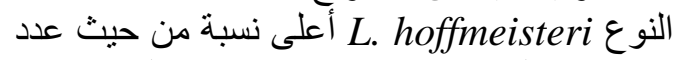

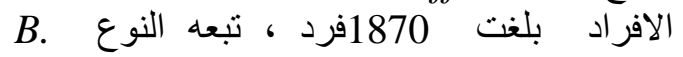
sowerbyi و L.profundicola الأنواع و L. udekemianus و L.claparedeianus 135 L. Lilavni

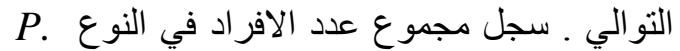
P. أعلى مما عليه في النوع النوع النو التي hammoniensis P. التوالي . وسجلت الانواع T. T. tubifex $M$. g R. coccienus و moravicus irroratus

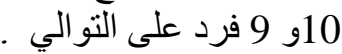

ومن حيث النسب المئوية لتكرار الانواع في الني B. L. Loffmeisteri العينات سجل النوان النوان sowerbyi

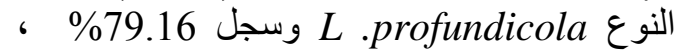

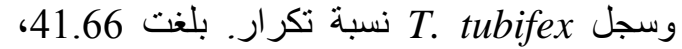

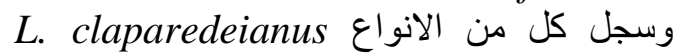
و النوع P. bavaricus نسبة تكرار 33. 33 \% \% P. وسجل النوعان hammoniensi

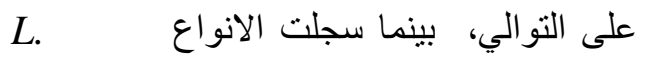
P. moravicusg R. coccienus, gsilvani و M. irroratus اوطأ نسب للتكرار في كل منهم $4.1 \%$ \% ( جدول 3).
Abundant أنواع وفيرة 40 - 70 \% species

Less 10 - 40 \% : أنواع قليلة وفرة abundant species Rare species أنواع نادرة 10

\section{النتائج والمناقشة: \\ يثير الجدول (1) الى القيم الدنيا و العليا}

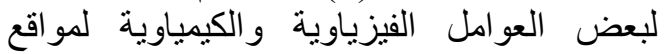

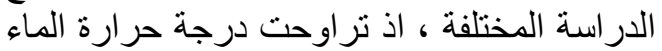

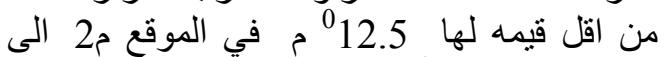

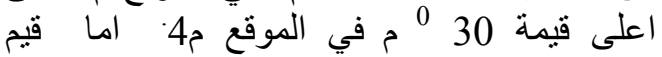

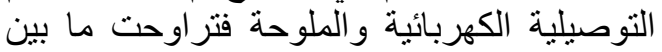
اقل القيم وبلغت.733 القية مايكروسيمنز اي مايعادل ملوحة 0.469 0\% في الموقع م4 ، و و وأعلى القيم

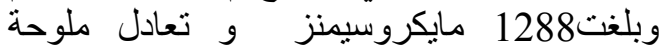

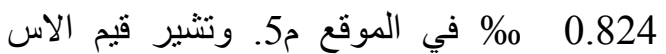

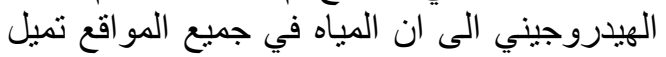

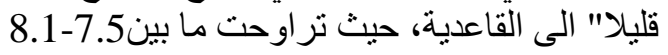

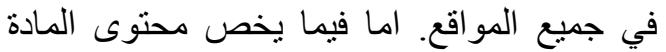

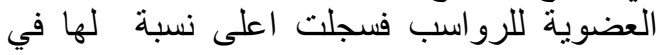

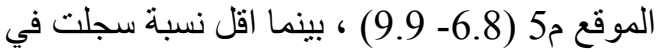

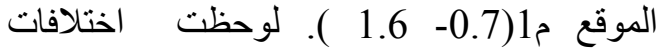
واضحة ل لنسجة القاع لمواقع الدراسة المختلفة

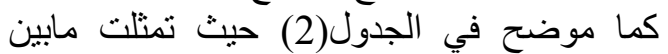
الطينية والغرينة و و الغرين الطينية والغرين المزيجية والغرين المزيجية ـ وفينة وفيما يخص الموقع الغرين م6 فتمايز بقاع غريني طيني مغطى التر بالنياتات

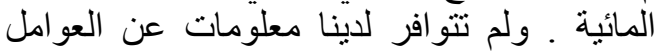
البيئية في هذا الموقع ـ لوحظة من خلائل الول نتائج

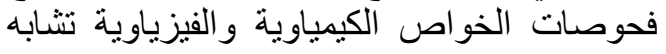

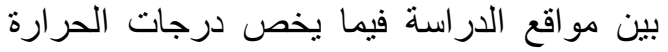

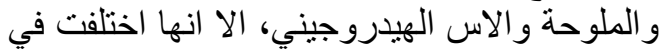
محتوى القاع من المادة العضوية التي كانت قليلة

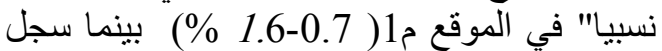

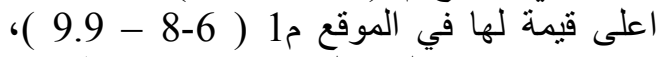

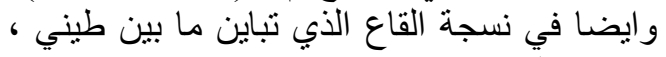

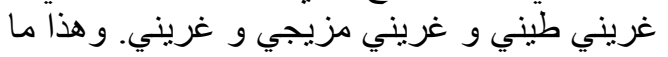

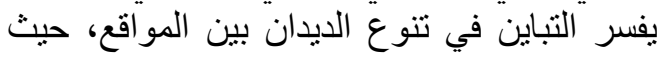

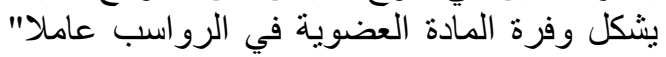

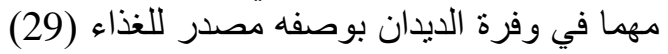

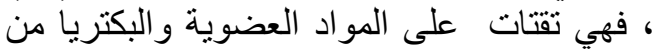

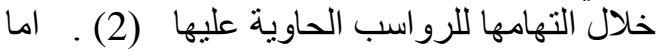

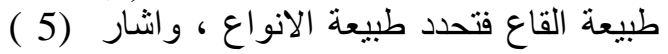

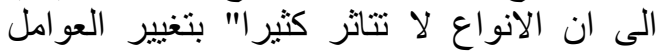

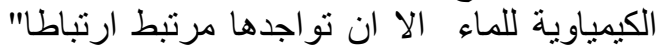

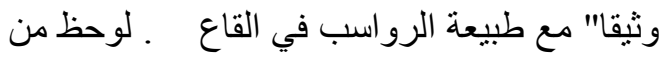

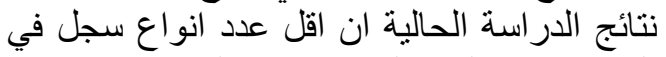
المواقع ذات القاع الطيني وهي الموقع م1 الع وم4 ، 


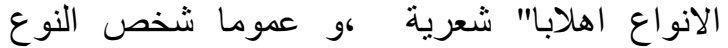
B. sowerbyi

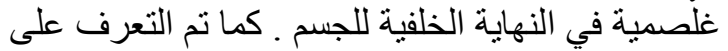

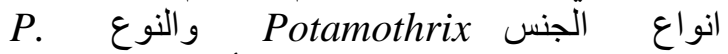
moravicus

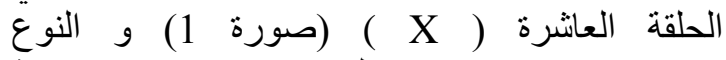
R.irroratus penial chaetae الجنس Limnodrilus فقد نم التقريق بينها من شكل الأن

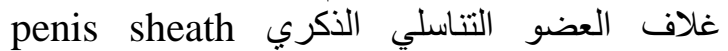
L. hoffmeisteri صورة 2) لوحظ سيادة النوع).

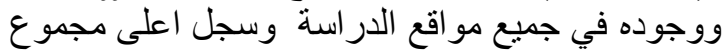

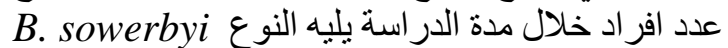

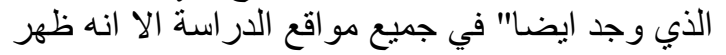

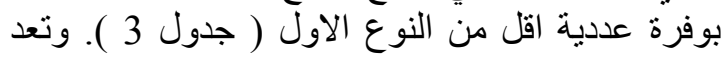

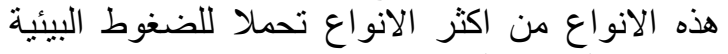

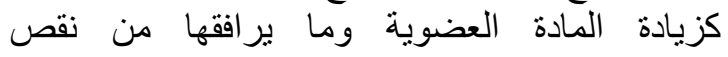

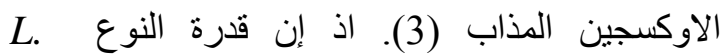
hoffmeisteri

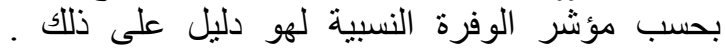

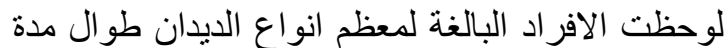

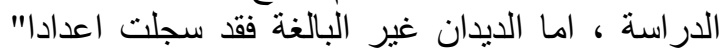

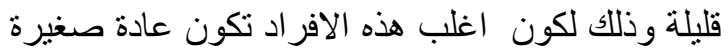

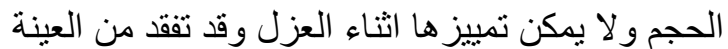

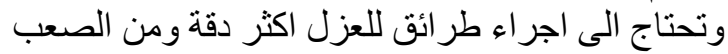

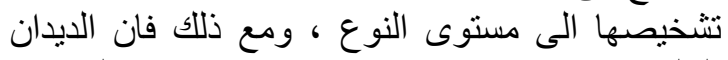

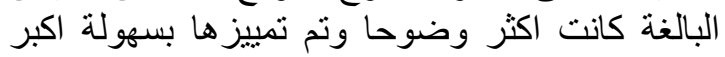

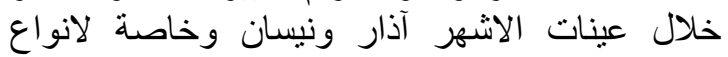

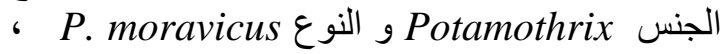

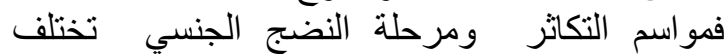

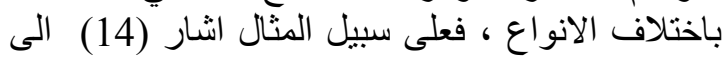

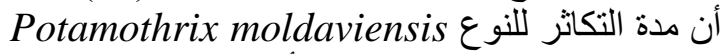
تعتمد على درجة الحرارة بشرط أن الن يكون الغذاء منو افر

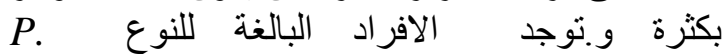
hammoniensis خلال فصل الربيع وبداية فصل الصيف (30) .
وفيما يخص عدد افراد الديدان المعزولة

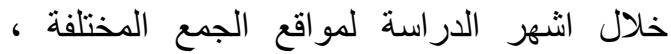

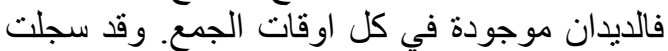

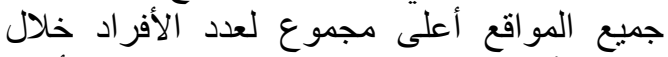

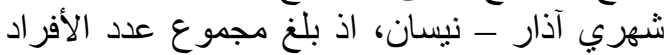

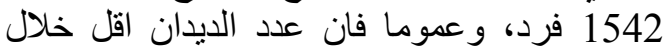

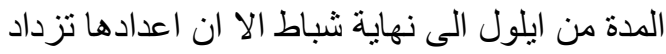

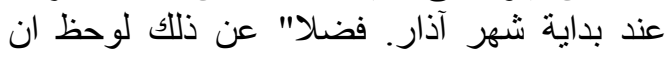

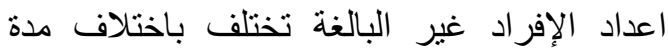

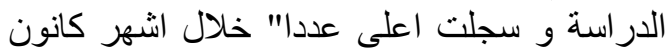
ثاني- شباط بلغ 58 فرد غير بالغ ، بينما سجل اقل التل

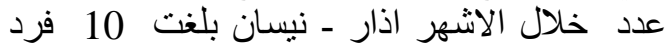

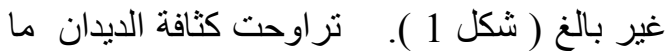

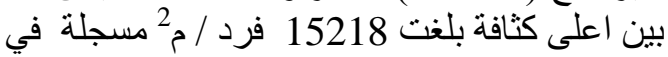

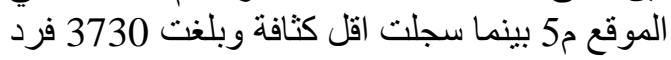

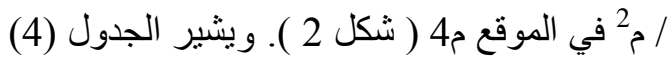

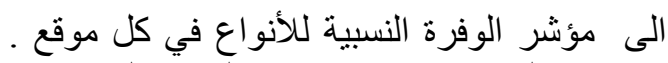

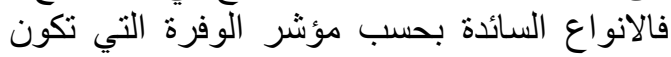

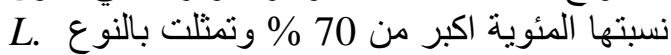

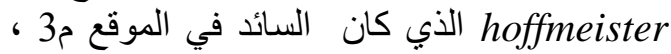

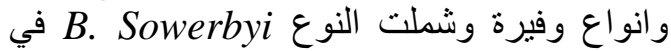

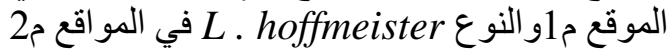
و م4 و م5و م6 ـ ـالما الانواع التي تنتراوح كثافاتها بين 10 - 40 \% من من الكثافة الكلية فتعد انواع قاع قليلة

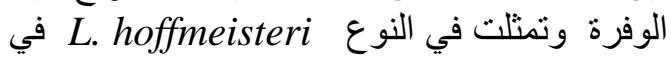

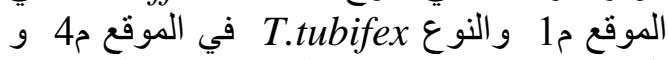

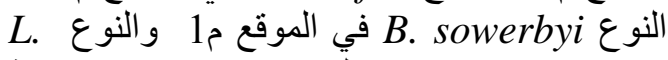
udekemianus

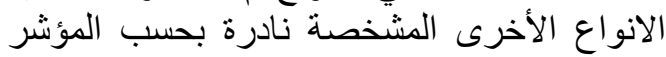

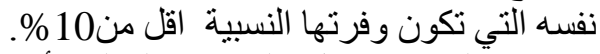

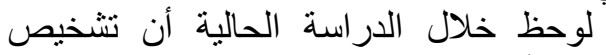

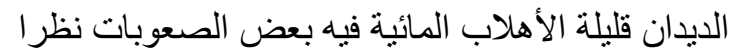

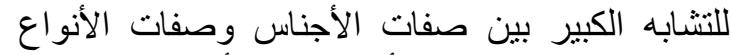

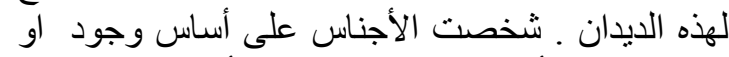

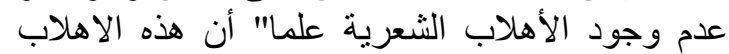

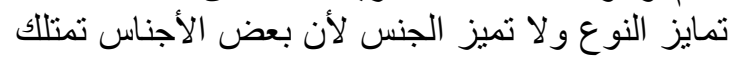

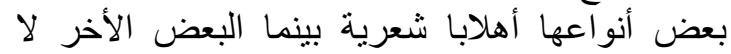

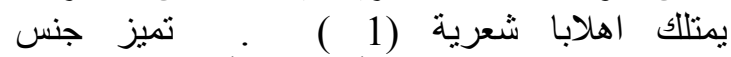
Limnodrilus

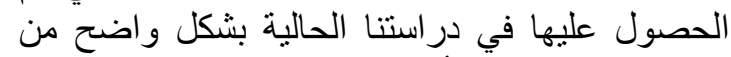
خلال عدم امتلاكه للأهلاب الثُعرية بينما امتلكت بقية 


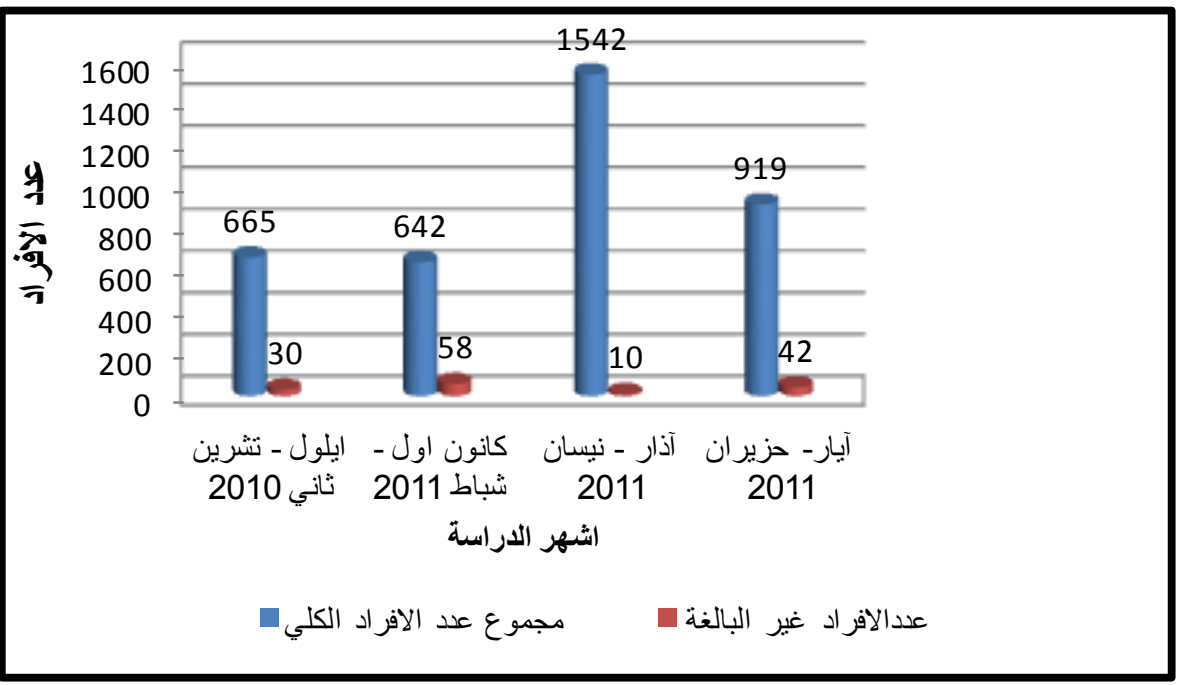

شكل 1: العدد الكلي و عدد الافراد غير البالغة لديدان تحت العائلة Tubificinae التي جمعت

خلال اوقات الدر اسة المختلفة.

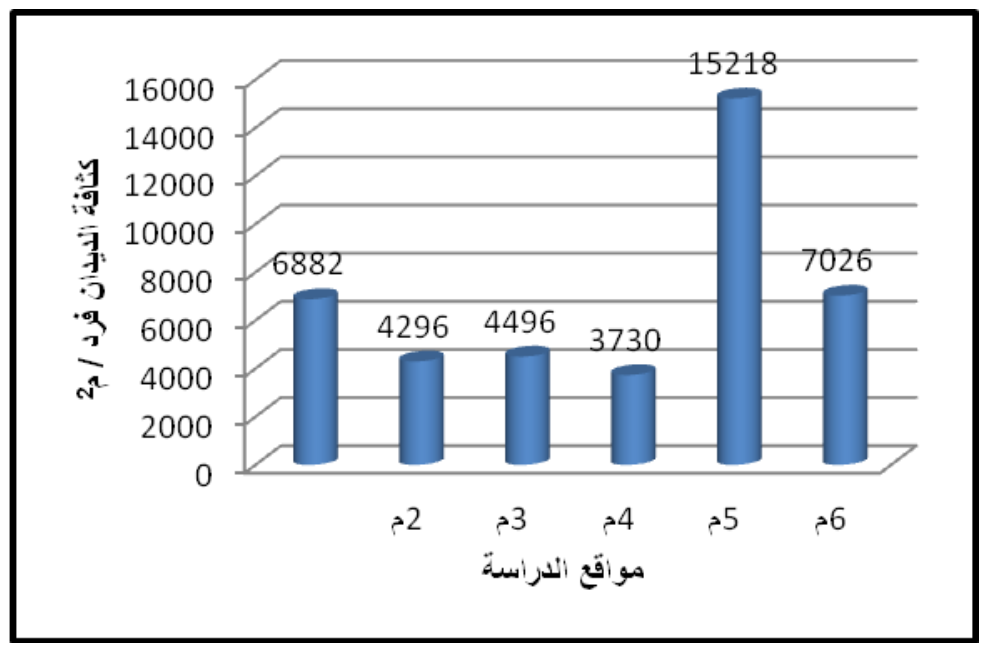

شكل (2): معدل كثافة الديدان / م² لجميع مناطق الدراسة

جدول (1) الحد الأعلى و الحد الأدنى للخو اص الكيماوية والفيزياوية و المادة العضوية لمو اقع الدراسة .

\begin{tabular}{|c|c|c|c|c|c|}
\hline العضوية & الأس & الملوحة \% & $\begin{array}{r}\text { (التوصيلية الكهربائية } \\
\text { (مايكروسمينز ) }\end{array}$ & المرجة حرارة & مواقع \\
\hline $1.6-0.7$ & $7.9-7.6$ & $0.797-0.459$ & $1245-753$ & $28-15$ & 12 \\
\hline $4.1-2.4$ & $8.1-7.9$ & $0.785-0.476$ & $1227-744$ & $27-12.5$ & 2 \\
\hline $3.2-1.3$ & $8.1-7.9$ & $0.785-0.475$ & $1227-742$ & $28-13.5$ & בג \\
\hline $3.9-1.6$ & $7.8-7.5$ & $0.787-0.469$ & $1227-733$ & $30-16$ & ב \\
\hline $9.9-6.8$ & $8.1-7.9$ & $0.824-0.459$ & $1288-753$ & $29-15.5$ & 5 \\
\hline
\end{tabular}


جدول(2) : نسجة القاع لمواقع الدراسة المختلفة.

\begin{tabular}{|c|c|c|c|c|}
\hline نسجة القاع & \% رمل & \% غرين & \% \%ين & مو اقعَّة \\
\hline طينية & 24.2 & 47.3 & 28.5 & م1 \\
\hline غرين طينية & 18 & 54.3 & 27.5 & ק2 \\
\hline غرين مزيجية & 65.4 & 22.1 & 12.5 & 3 \\
\hline طينية & 28.6 & 48.8 & 23 & ק4 \\
\hline غرينية & 18.6 & 58.4 & 23 & ק \\
\hline غرين طينية & 18 & 54.3 & 27.5 & $\sigma_{p}$ \\
\hline
\end{tabular}

جدول (3) : عدد الافر اد المشخصة من ديدان تحت العائلة Tubificidae التي جمعت من مو اقع الدر اسة و نسبها المئوية والنسبة المئوية لتكر ار كل نوع في عينات الآدر اسة .

\begin{tabular}{|c|c|c|c|c|c|c|c|c|c|}
\hline المواقع & م1 & $2 \beta$ & 3 & م4 & ק & ק & 亨. & 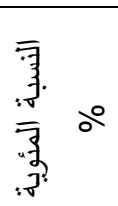 & $\begin{array}{l}\stackrel{\overline{3}}{7} \\
\overbrace{}^{\circ}\end{array}$ \\
\hline $\begin{array}{l}\text { Limnodrilus } \\
\text { hoffmeisteri }\end{array}$ & 202 & 216 & 266 & 198 & 675 & 313 & 1870 & 52 & 100 \\
\hline L. profundicola & 15 & 23 & 16 & 17 & 58 & 6 & 135 & 4 & 79.16 \\
\hline L. claparedeianus & - & 9 & - & - & 37 & 5 & 51 & 1.40 & 33.33 \\
\hline L.udekemianus & - & 9 & - & - & 355 & - & 364 & 10.03 & 29.16 \\
\hline L.silvani & - & - & - & - & - & 13 & 13 & 0.35 & 4.16 \\
\hline Tubifex tubifex & - & 20 & - & 39 & - & 42 & 101 & 3 & 41.66 \\
\hline $\begin{array}{r}\text { Potamothrix } \\
\text { bavaricus }\end{array}$ & - & 22 & 35 & - & 6 & - & 63 & 2 & 33.33 \\
\hline P. hammoniensis & - & - & - & - & 54 & - & 54 & 1.48 & 12.5 \\
\hline $\begin{array}{r}\text { Psammoryctides } \\
\text { moravicus }\end{array}$ & - & 12 & - & - & - & - & 12 & 0.33 & 4.16 \\
\hline $\begin{array}{r}\text { Monopylephorus } \\
\text { irroratus }\end{array}$ & - & - & - & - & 9 & - & 9 & 0.24 & 4.16 \\
\hline $\begin{array}{r}\text { Rhyacodrilus } \\
\text { coccineus }\end{array}$ & - & - & 10 & - & - & - & 10 & 0.27 & 4.16 \\
\hline $\begin{array}{r}\text { Branchiura } \\
\text { sowerbyi }\end{array}$ & 389 & 57 & 58 & 72 & 152 & 218 & 946 & 26.07 & 100 \\
\hline مجموع الأفراد & 606 & 368 & 385 & 326 & 1346 & 597 & 3628 & & \\
\hline عدد الأنواع & 3 & 8 & 5 & 4 & 8 & 6 & & & \\
\hline
\end{tabular}


جدول (4 ): توزيع أنواع تحت العائلة Tubificinae ووفرتها النسبية بحسب مؤشر الوفرة النسبية في مواقع الدراسة المختلفة . (Ra)

\begin{tabular}{|c|c|c|c|c|c|c|}
\hline & & & & & \multicolumn{2}{|c|}{ مواقع الدراسـة } \\
\hline & م & 2 & م 3 & م & م & م6 \\
\hline Limnodrilus hoffmeisteri & ++ & +++ & ++++ & +++ & +++ & +++ \\
\hline L. profundicola & + & + & + & + & + & + \\
\hline L. claparedeianus & - & + & - & - & + & + \\
\hline L.udekemianus & - & + & - & - & ++ & - \\
\hline L.silvani & - & - & - & - & - & + \\
\hline Tubifex tubifex & - & + & - & ++ & - & + \\
\hline Potamothrix bavaricus & - & + & + & - & - & - \\
\hline P. hammoniensis & - & - & - & - & + & - \\
\hline $\begin{array}{r}\text { Psammoryctides } \\
\text { moravicus }\end{array}$ & - & + & - & - & + & - \\
\hline Monopylephorus irroratus & - & - & - & - & + & - \\
\hline Rhyacodrilus coccineus & - & - & + & - & - & - \\
\hline Branchiura sowerbyi & +++ & ++ & ++ & ++ & ++ & ++ \\
\hline ديدان غير بالغة & + & + & + & + & + & + \\
\hline
\end{tabular}

++++أنواع سائدة ، D اكبر من 70\% ، +++ أنواع وفيرة ، D تتراوح مابين 40 - 70 \% ، ++ أنواع قليلة ، D تتراوح مابين

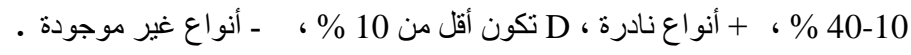




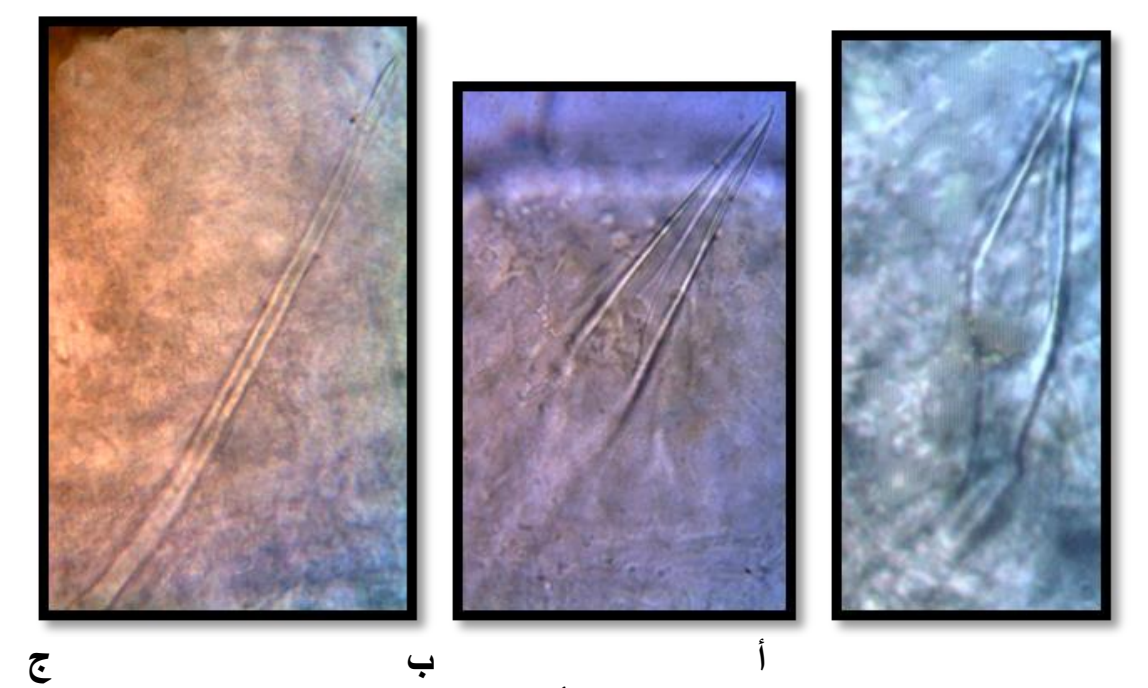

صورة 1: اهلاب اكياس استقبال النطف: أ: النوع Potamothrix bavaricus ، ب: النوع . P. hammoniensis ج: النوع Pammoryctides maravicus

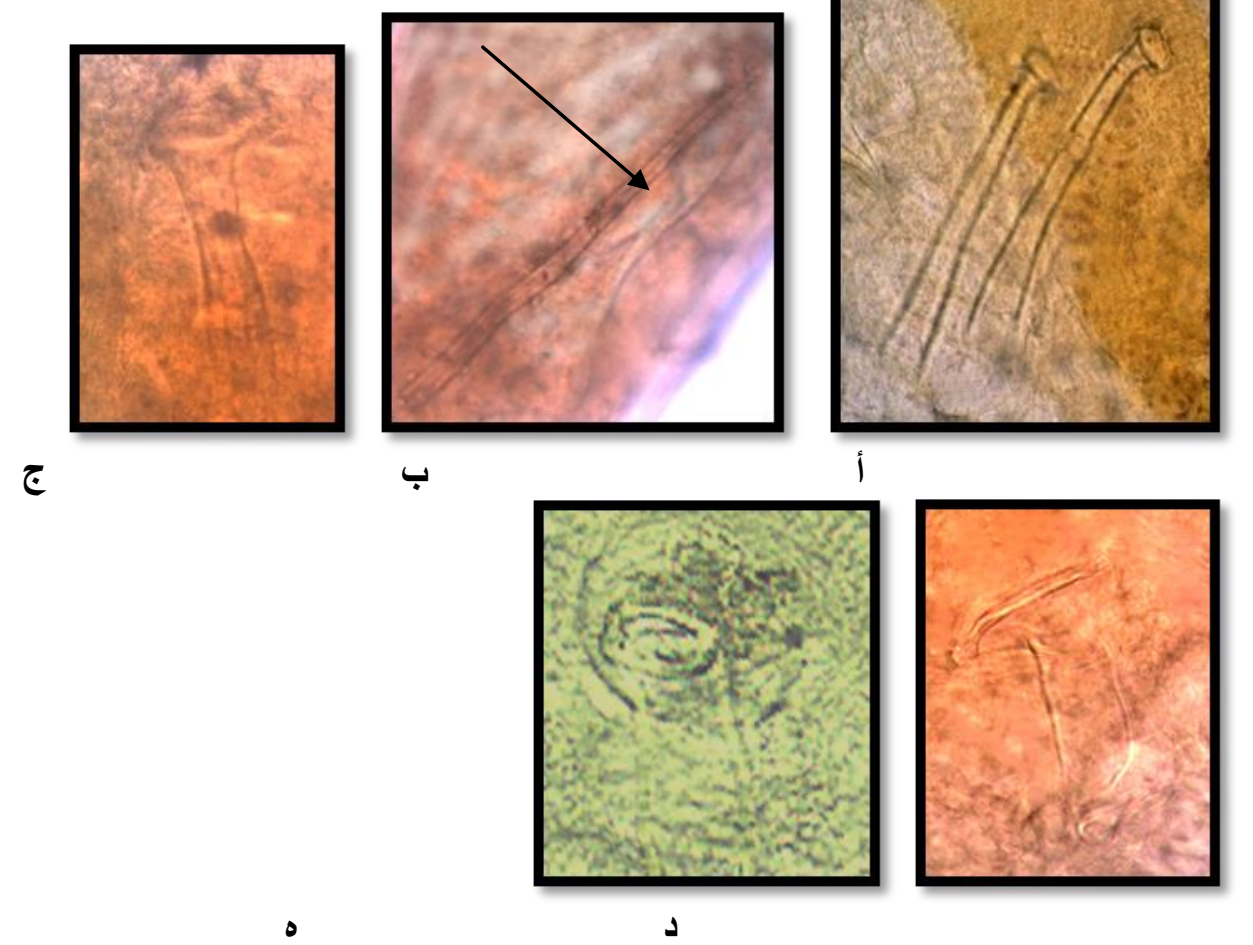

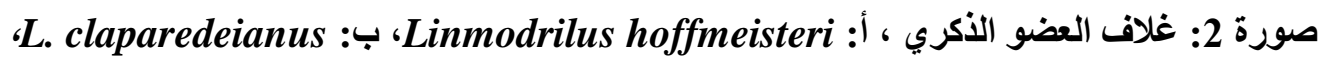

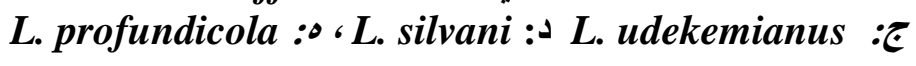




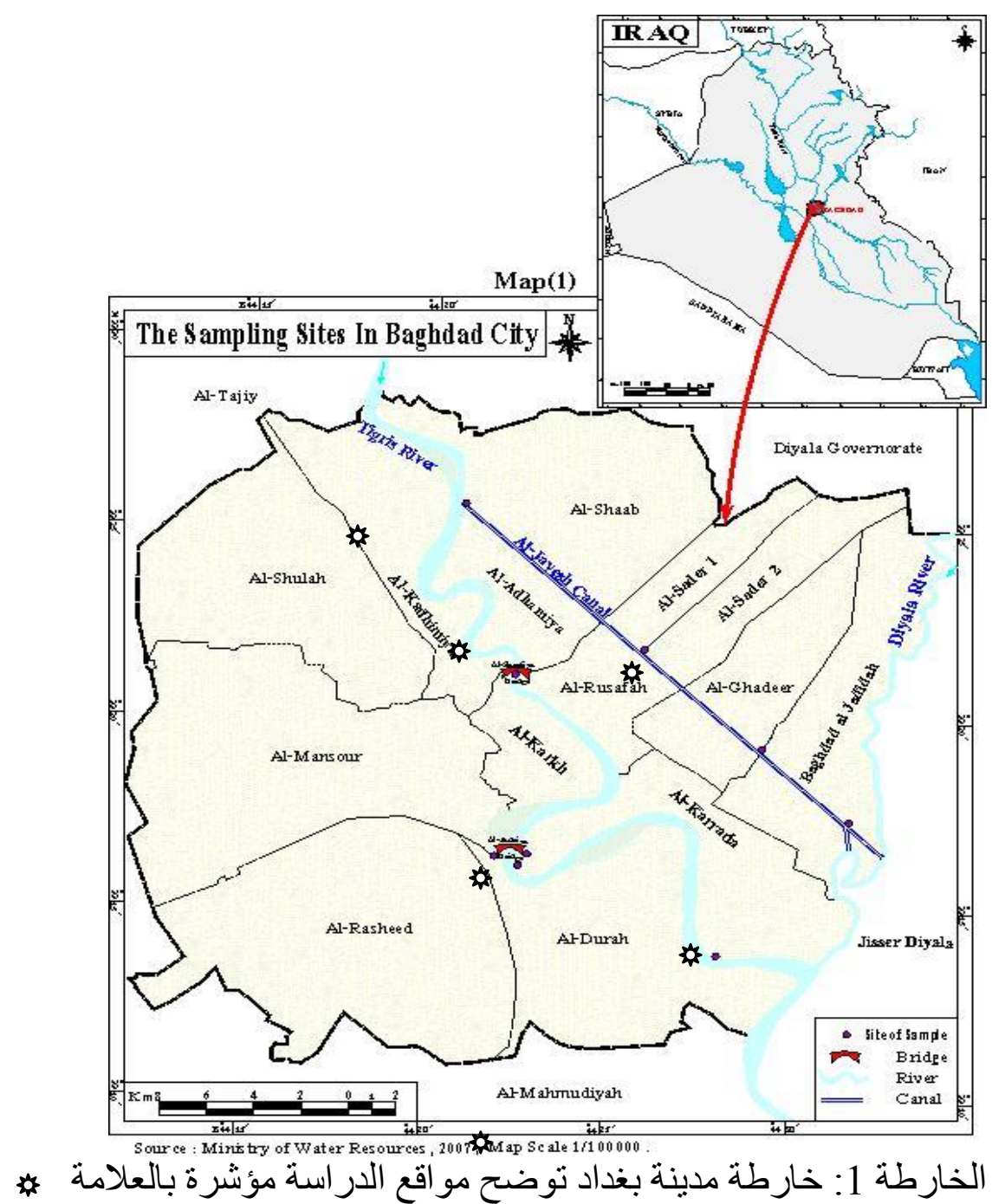

4- Petridis, D. and Sinis, A. 1997 The benthic fauna of lake Mikri Prespa. Hydrobiologia , 351: 95-105.

5- Timm , T., Seire , A. and Pall , P. 2001 Half acentury of oligochaete research in Estonian

running waters . Hydrobiologia , 463 : 223- 234.

6- Timm, T2009 A guide to the freshwater Oligochaeta and Polychaeta of Northern and Central Europe. Lauterborina, 66:1235

7- Erséus, C., Prestegaard, T., and Källersjö, M. 2000 Phylogenetic analysis of the Tubificidae

(Annelida, Clitellata) based on $18 \mathrm{~S}$ rDNA sequences. Molecular

1- Brinkhurst, R.O. and Jamieson, B.G.M1971 Aquatic Oligochaeta of the world. Univ. of Toronto Press. Toronto, Canada. 860 pp.

2- Swayne , H. , Day , M. and Wetzel , M. J. 2004 . Limnodrilus hoffmeisteri ( Annelida: Oligochaeta Tubificidae ) in Pop 's cave, Wisconsin, U S A . Journal of cave and Karst studies , 66 (1) : 28- 31.

3- Kazanci , N. and Girgin , S. 1998 Distribution of oligochaetes species as bioindicators of organic pollution in Akara Stream and their use in biomonitoring . Turk. J. Zool. , $22: 83-87$. 
The benthic Fauna of the Polluted lower Part of river Diyala, Central Iraq. JBSR . Vol. 17(3) :35-45.

17- Al-Lami , A. A. , Jaweir, H. J. , \& Nusha'at, M. R. 1997. Benthic invertebrates community of river Ephrates Upstream and downstream sectors of AlQadisia Dam , Iraq . Regulated river , Research and Management , 14(4) : 383-390.

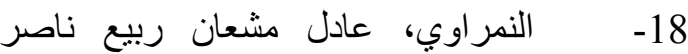
2005 و اللافقريات

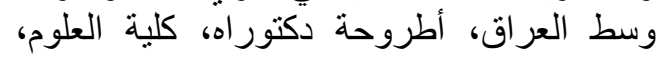

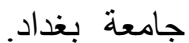

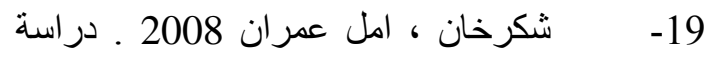
بيئية لمجتمع اللافقريات القاعية لنهر الفرات في النيات

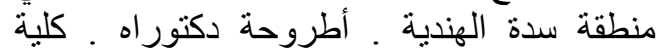
العلوم ـ الجامعة المستنصرية .

20- Kassim, T.I.; Jaweir, H.J.; Muften, F.S., Al-Maliky, S.K. and Nashaat, M.R. 1997 Benthic fauna in Tigris River, Iraq. J.Coll. Educ. For Women, Univ. Baghdad, 8 (2): 167170.

21- Nashaat, M.R., Al-Lami, A.A. Jaweir, H.J. 2000 Benthic fauna of Qadisia Lake, North west Iraq. J.Diala 8(1): 74-83

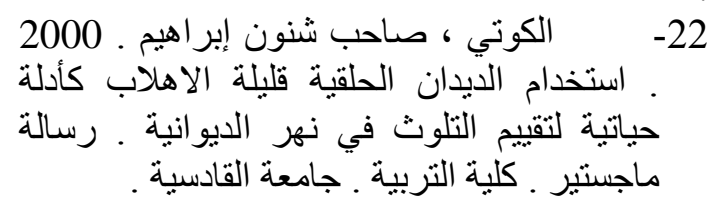

23- Ali, L.A 2007 A study of macroinvertebrates community in the middle sector of Greater Zab River/Iraq. Ph.D. thesis, Colloge of Science for Women, Baghdad Univ., 123pp.

24- Sabtie, H.A. 2009 An ecological study of the benthic macroinvertebrates community in the Southern Marshes of Iraq. Ph.D.thesis, Colloge of Science for
Phylogenetics and Evolution, 15: 381-389.

8- Erséus, C., Källersjö, M., Ekman, M. and Hovmöller, R . 2002 18S rDNA phylogeny of the Tubificidae (Clitellata) and its constituent taxa: dismissal of the Naididae. Molecular

Phylogenetics and Evolution, 22: 414-422.

9- Erséus, C. 2005 Phylogeny of oligochaetous Clitellata. Hydrobiologia, $\quad 535 / 536$ : $357-$ 372.

10- Erséus, C., Wetzel, M.J. and Gustavsson, L. 2008ICZN rules-a farewell toTubificidae (Annelida, Clitellata). Zootaxa, 1744: 66- 68.

11- Paoletti , A1989. Cohort cultures of Tubifex tubifex forms . in J. L. Kaster (ed) , Aquatic Oligochaete Biology. Kluwer Academic publisher, Belgium . Hydrobiologia , 180:143-150.

جوير ، هيفاء جواد ، الدركزلي ، منال $-12$

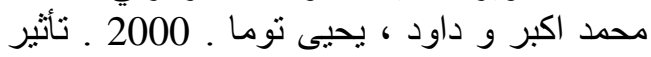
Tubifex tubifex نوع الغذاء على إنتاجية النوع دانير ديدان (Oligochaeta : Tubificidae ) حلقية - قليلة الاهلاب في الأوساط الزراعية التجريبية ـ مجلة كلية التربية للبنات . جامعة بغداد 11 ( 2 ) : 46 - 46 .

13- Verdonschot , P. F. M. 2006. Beyond masses and blooms : the indicative value of oligochaetes . Hydrobiologia, $564: 127-142$.

14- Milbrink, G, Timm , T. and Lundberg , S. 2002. Indicative profundal Oligochaete assemblages in selected small Swedish Lakes . Hydrobiologia ,468: 53-61.

15- Lietz, D. M. 1987 Potential for aquatic oligochaetes as live food in commercial aquaculture.

Hydrobiologia, 155: 309-310.

16- Al-Mukhtar, E. A. AlDabbagh, and Taha, T.M. 1986 
28- Omori, M. and Ikeda,T1984 Methods in marine zooplankton ecology. Wiley and Sons,New York

29- Peralta, L., Escobar , E. , Alcocer , J. and Lugo , A. 2002 Oligochaetes from six tropical crater lakes in Central Mexico species composition, density and biomass. Hydrobiologia , $467: 109-116$.

30- Arslan, N. and Şahin Y. 2006. A preliminary study on the Identification of the Littoral Oligochaete ( Annelida ) and Chironomidae (Diptera) Fauna of Lake Kovada , a National Park in Turkey. , Turky. J. Zool., 30: 67-72.
Women , Baghdad Univ. Baghdad, Iraq

25- Nashaat, M.R. 2010 Impact of AL-Durah Power Plant effluents on physical, chemical and invertebrates biodiversity in Tigris River, Southern Baghdad. Ph. D. thesis, College of Science, Baghdad Univ.

26- Jaweir, H.J. 2012 . A new record of three tubificid species ( Annelida : Oligochaeta ) from Alhaweizah marsh. Iraq. Mesopot. J. Mar. Sci. 26(2) : 114- 121.

27- $\quad$ Brinkhurst, R.O. 1971 A guid for the identification of British aquatic Oligochaeta.

Freshwater Biological Association, Scientific publication $\quad$ No:2

\title{
Sludge worms species (Oligochaeta: Naididae: Tubificinae) From Different Aquatic Habitat in Baghdad / Iraq
}

\author{
Haifa J. Jaweir* \\ Afrah M. Alwan* \\ *Department of Biology, College of Science for Women \\ University of Baghdad
}

\begin{abstract}
:
The present investigation is concerned primarily with sorting and identification of subfamily Tubificinae from River Tigris and some water surface in Baghdad /Iraq . For this purpose six study sites were chosen, including a Al- Kadhemyia drainage canal, North Baghdad; three sites on the shore of River Tigris; in addition to site in Al-Jaish canal, East Baghdad, finally the sixth site was from pond in Al-Zawra'a park , within the center of Baghdad .These sites were characterized by sediment rang from clay, silty-clay to silty with a percentage of organic matter ranged between $0.7 \%$ $9.9 \%$. According to water temperature, salinity and $\mathrm{pH}$ values, they were nearly identical in all study sites, the water temperature was ranged through the study period between $12.5-30^{\circ} \mathrm{C}$, while electrical conductivity and salinity values were ranged beteen 733.5-1227.5 $\mu \mathrm{s}$, which equivalent to 0.469-0.787 \%o salinity. All sites were slightly alkaline, so the $\mathrm{pH}$ values were ranged between 7.5-8.1.

3628 individuals were sorted from 24 samples collected from different study sites.. Twelve species were identified belonging to seven genera, including, Limnodrilus hoffmeisteri, L. claparedeianus, .L. profundicola, . L.udekemianus, L. silvani, Tubifex tubifex, Branchiura sowerbyi, Potamothrix.
\end{abstract}


bavaricus, , P. hammoniensis, Psammoryctide moravicus, Monopylephorus irroratus, and Rhyacodrilus coccineus.

The result revealed that Al-Jaish canal and Tigris River / al-Sarafyia were considered as the richest sites according to the number of species recorded, where 8 species were recorded in each of them, but Al-Jaish canal had higher individual number of 1346 individuals, followed by Al-Kadhemyia drainage canal recorded only three species with high total individual number of 606 individual. The lowest total individual number was recorded in Tigris River / Al- Zaafaranyia of 326 individual.

L. hoffmeisteri, records higher individuals number of 1870 individual , followed by $B$. sowerbyi which records 946 individual. Both species recorded highest percentage occurance of $100 \%$ followed by $79.16 \%$ recorded by $L$. profundicola.

High densities of Tubificinae were recorded during all study period. The highest density was during March-April, reached between $2109-534.9 \mathrm{ind} / \mathrm{m}^{2}$ in Tigris River sites , $7270.5 \mathrm{ind} / \mathrm{m}^{2}$ in Al-Jaish canal and $2419.8 \mathrm{ind} / \mathrm{m}^{2}$ in Al-Zawra'a pond. The results of relative abundance revealed that L. hoffmeisteri was the dominant species in site Tigris River/Al- Jaderyia, and abundant in other study sites. $B$. sowerbyi was abundant in all study sites, while other species were recorded between few to rare species except L. udekemianus which was abundant in site Al-Jaish canal, and T. tubifex in site Tigris River / Al- Zaafaranyia. 